n.tronos

glyndwhr

Glyndŵr University

Glyndŵr University Research Online

Computing

Computer Science

$1-1-2005$

\title{
Computation of specific absorption rate in the human body due to base-station antennas using a hybrid formulation
}

\author{
RA. Abd-Alhameed \\ Peter S. Excell \\ Glyndwr University, p.excell@glyndwr.ac.uk \\ M A. Mangoud
}

Follow this and additional works at: http://epubs.glyndwr.ac.uk/cair

Part of the Computer Engineering Commons, and the Computer Sciences Commons

\section{Recommended Citation}

Abd- Alhameed, R. A., Excell, P. S., \& Mangoud, M. A. (2005)'Computation of specific absorption rate in the human body due to base-station antennas using a hybrid formulation'. IEEE Transactions on Electromagnetic Compatibility, 47(2), 374-381.

This Article is brought to you for free and open access by the Computer Science at Glyndŵr University Research Online. It has been accepted for inclusion in Computing by an authorized administrator of Glyndŵr University Research Online. For more information, please contact

d.jepson@glyndwr.ac.uk. 


\title{
Computation of specific absorption rate in the human body due to base- station antennas using a hybrid formulation
}

\begin{abstract}
A procedure for computational dosimetry to verify safety standards compliance of mobile communications base stations is presented. Compared with the traditional power density method, a procedure based on more rigorous physics was devised, requiring computation or measurement of the specific absorption rate (SAR) within the biological tissue of a person at an arbitrary distance. This uses a hybrid methd of moments/finite difference time domain(MoM/FDTD) numerical method in order to determine the field or SAR distribution in complex penetrable media, without the computational penalties that would result from a wholly FDTD simulation. It is shown that the transmitted power allowed by the more precise SAR method is, in many cases, between two and five times greater than that allowed by standards implementing the power flux density method.
\end{abstract}

\section{Keywords}

Hybrid method, specific absorption rate (SAR), safety standards, computational dosimetry

\author{
Disciplines \\ Computer Engineering | Computer Sciences
}

\section{Comments}

(C)2005 IEEE. Personal use of this material is permitted. However, permission to reprint/republish this material for advertising or promotional purposes or for creating new collective works for resale or redistribution to servers or lists, or to reuse any copyrighted component of this work in other works must be obtained from the IEEE. This material is presented to ensure timely dissemination of scholarly and technical work. Copyright and all rights therein are retained by authors or by other copyright holders. All persons copying this information are expected to adhere to the terms and constraints invoked by each author's copyright. In most cases, these works may not be reposted without the explicit permission of the copyright holder. This paper was published in IEEE Transactions on Electromagnetic Compatibility in 2005. The definitive version is available at http://ieeexplore.ieee.org 


\title{
Computation of Specific Absorption Rate in the Human Body due to Base-Station Antennas Using a Hybrid Formulation
}

\author{
Raed A. Abd-Alhameed, Peter S. Excell, Senior Member, IEEE, and Mohab A. Mangoud, Member, IEEE
}

\begin{abstract}
A procedure for computational dosimetry to verify safety standards compliance of mobile communications base stations is presented. Compared with the traditional power density method, a procedure based on more rigorous physics was devised, requiring computation or measurement of the specific absorption rate (SAR) within the biological tissue of a person at an arbitrary distance. This uses a hybrid methd of moments/finite difference time domain (MoM/FDTD) numerical method in order to determine the field or SAR distribution in complex penetrable media, without the computational penalties that would result from a wholly FDTD simulation. It is shown that the transmitted power allowed by the more precise SAR method is, in many cases, between two and five times greater than that allowed by standards implementing the power flux density method.
\end{abstract}

Index Terms-Hybrid method, specific absorption rate (SAR).

\section{INTRODUCTION}

$\mathbf{T}$ HE traditional method for assessment of the compliance of fixed radio transmitters with safety guidelines is to calculate the radiated power flux density (PFD) in Watts per square meter at the minimum distance of approach by personnel and to compare this with the maximum values that are specified in many safety guideline documents [1], [2]. The calculation of PFD may be undertaken using simple far-field power-based relationships or by a more accurate computational-electromagnetics method that predicts electric field strength in arbitrary directions and distances, converting this to $\operatorname{PFD}(S)$ through the standard relation $S=E^{2} / Z_{0}$.

The PFD procedure is not viable for hand-held transmitters which couple to the biological tissue in the near field and for this situation a procedure based on more rigorous physics is used, requiring computation or measurement of the specific absorption rate (SAR) within the biological tissue. This method is thus directly related to the rate of deposition of the heat energy in the tissue. This approach requires detailed computational models if it is to be assessed predictively, but such techniques have now become well-established [3].

It is thus desirable that the techniques developed for compliance assessment based on SAR be extended to cover transmitters further from the human head than is normal for mo-

Manuscript received May 15, 2002; revised August 31, 2004.

R. A. Abd-Alhameed and P. S. Excell are with the Mobile and Satellite Communications Research Centre, University of Bradford, Bradford BD7 1DP, U.K. (e-mail: r.a.a.abd@brad.ac.uk; p.s.excell@brad.ac.uk).

M. A. Mangoud is with the Arab Academy for Science and Technology, Alexandria 1029, Egypt. (e-mail: mangoud@aast.edu).

Digital Object Identifier 10.1109/TEMC.2005.847395 bile handsets. Unfortunately, if the finite difference time domain method is used to simulate this, the size of the computational problem becomes excessive when the transmitter is further than a modest distance from the body. An alternative is to use an integral equation method such as the method of moments (MoM). This will model the field from the antenna at arbitrary distances with good accuracy, but the size of the computational task becomes unrealistic if it is used to represent the detailed structure of the human head.

A solution to this problem is to use a computer program that is a hybrid of finite difference time domain (FDTD) and MoM such that the more appropriate of these two methods can be applied to the different parts of the problem. A hybrid MoM/FDTD method may be said to have been first investigated in 1982, when Taflove and Umashankar [4] used a hybrid FDTD/MoM approach to investigate coupling problems and aperture penetration into complex geometries and loaded cavities, for example a missile guidance section. This used MoM to solve the exterior problem and FDTD to model complex interior problems. The two regions were linked via an equivalent short-circuit electric current excitation in the aperture regions of the structure. Unlike the present work, it did not employ computations of equivalent magnetic current on a virtual equivalent surface and it was only intended for field penetration problems into a closed cavity region. In 1987, the same authors [5] used an equivalent surface fully enclosing wire bundles to replace them with a single wire in the FDTD model. Later, the concepts in [4] and [5] were deployed in the program GEMACS [6] which used MoM/uniform theory of diffraction (UTD)/finite difference frequency domain (FDFD) hybrids to model problems with multiple regions. FDFD was used to model the interior region(s) only.

More recently, Bretones et al. presented [7] a time-domain version of MoM in a hybrid approach for studying the transient excitation of a thin wire antenna located in the proximity of an inhomogeneous dielectric scatterer and above a perfectly electrically conducting ground plane. Also, Cerri et al. [8] used a time-domain version of MoM for a hybrid technique [8]. These methods have the advantage of generating information over a wide frequency band. They do not require an iterative procedure to couple with FDTD, but they require large run-times when treating a junction with more than two wires [9]. In contrast, the frequency-domain version used here allows complex metallic structures to be modeled accurately in modest run-times and with more flexibility for modeling different complex geometries. This is aided by the greater maturity, flexibility and industrial acceptance of frequency-domain MoM 
programs, due to the longer period over which they have been evolving. Huang et al. [10] employed a hybrid technique for modeling the interaction of ground-penetrating radar (GPR) with complex ground using a combination of frequency domain MoM, Fourier transformation and iterations. This method has the same principles as the method proposed in the present work, while it focuses on GPR applications (the project started independently of, and approximately contemporaneously with, the present work [18]. The principal differences are in the applied MoM versions and in the implementation procedures). Recently, another hybrid MoM/FDTD method [11] was applied for numerical simulations of SAR and the magnetic field of shielded RF coils loaded with a human head for a biomedical application. In [12] the source antenna is modeled as a stack of Hertzian dipoles. However, in [11] and [12], the authors neglect the effect of the back-scattered field on the source. The same approximation is used in [13] which is oriented toward two-dimensional UHF/VHF propagation problems.

Research into these methods is intensifying and more groups have become interested. Rubio Bretones et al. have recently published a method to combine the numerical electromagnetic code (NEC) MoM program with FDTD [14]. However, the chosen method for calculation of the back-scattered field on the wire results in long run times as the algorithm entails running the FDTD code $N_{s}$ times (where $N_{s}$ is the number of the basis functions on the wire antenna): this is avoided in the work presented below. Some interesting comparisons between the MoM and FDTD numerical methods are published in [15] for modeling electrically small antennas and in [16] for radiation and scattering involving dielectric objects. The advantages of each technique are discussed: this shows how the hybrid method is a promising and effective technique, being able to exploit the strengths of both methods.

The combination of FDTD and frequency-domain MoM that is extended in the present work was originally developed for the case of a mobile phone adjacent to a human head [17]-[21]. When modeling a mobile phone, the MoM region is kept as small as possible, just sufficient to contain the phone itself and simulate its complex (e.g., helical) antenna. This region is placed as a subdomain within the FDTD region and surrounded by a pair of bounding surfaces (a Huygens surface and an equivalence-principle surface) which are used to relaunch the inward and outward travelling fields between the two domains.

For studies of the effect of base stations on the body, the size of the moment-method region would be too large for it to be treated as a subdomain, and hence a modified formulation was devised, essentially treating the FDTD region as the subdomain. To achieve correct conversion between the frequency and time domains, simple Fourier transforms (monochromatic, including phase information) are applied between each iterative step, in which interim field data is transferred between MoM and FDTD. The induced currents for the source region are first obtained, excluding the effect of the scatterer in the FDTD region, using the frequency domain version of MoM. The fields due to these currents are obtained on the closed surface (Huygens surface) [22] that separates the source from the scatterer. Oscillating with respect to a reference phase of the source, these fields or their equivalent surface currents are converted to time-domain inci-

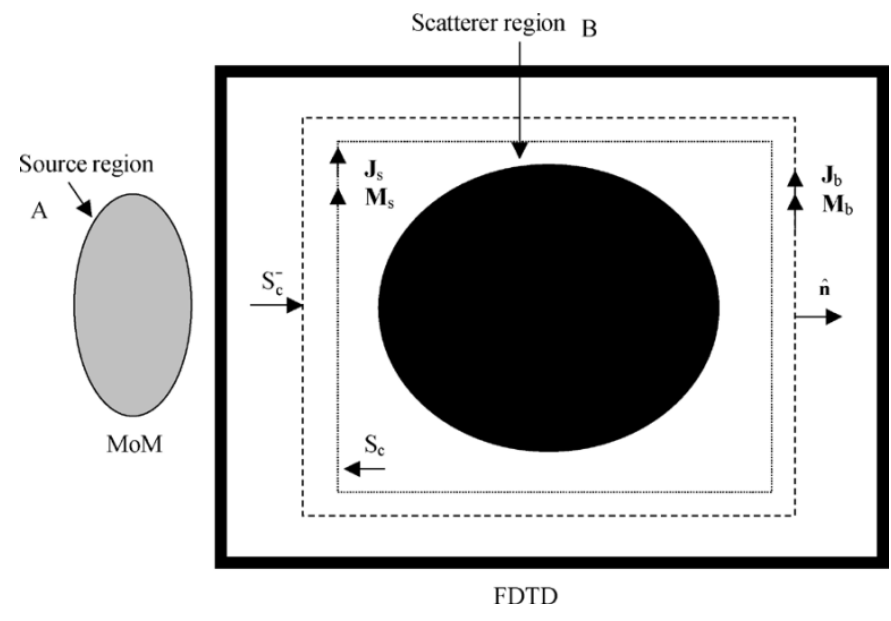

Fig. 1. Generic example of MoM source region and FDTD scatterer subregion.

dent fields or current sources using an inverse discrete Fourier transform. The FDTD algorithm is now executed with these time-domain sources to obtain the induced currents on the scatterer. The back-scattered fields on the source side of the Huygens surface become supplementary excitations for the source region. These fields or their equivalent current sources are transferred to the frequency domain using a discrete Fourier transform, in which the phase difference relative to the reference phase of the source is taken into account. The MoM model is then rerun to evaluate the induced currents on the source region due to both the source excitation region and the induced equivalent current sources from the FDTD method. The method is repeated until a steady-state solution is obtained.

\section{THEORY OF THE METHOD}

Firstly, consider Fig. 1, which shows a source region and a scatterer subregion. The scatterer region is bounded by the closed surface $S_{c}$, on which the equivalence principle will be applied. The method starts by applying the MoM for the source (assuming internal excitation exists in the source region). The fields on the equivalence-principle surface $S_{c}$ can then be computed. Thus the excitation surface currents that are required to run the FDTD, including the scatterer region, can be evaluated as follows:

$$
\begin{aligned}
\mathbf{M}_{s} & =-\hat{\mathbf{n}} \times \mathbf{E}_{s}\left(S_{c}\right) \\
\mathbf{J}_{s} & =\hat{\mathbf{n}} \times \mathbf{H}_{s}\left(S_{c}\right)
\end{aligned}
$$

where $\mathbf{E}_{s}$ and $\mathbf{H}_{s}$ are the scattered electric and magnetic fields on the surface $S_{c} . \mathbf{J}_{s}$ and $\mathbf{M}_{s}$ are the equivalent surface currents on the same surface and $\hat{\mathbf{n}}$ is the unit vector directed outwards from the (closed) surface.

Thus these currents are treated as the source in the FDTD domain, propagating fields to the scatterer by using the $E$ and $H$ time-domain equations. Thus, the time-domain equations on the surface $S_{c}$ can be stated as follows:

$$
\begin{aligned}
& \nabla \times \mathbf{E}=-\frac{\partial \mathbf{B}}{\partial t}-\mathbf{M}_{s} \\
& \nabla \times \mathbf{H}=\frac{\partial \mathbf{D}}{\partial t}+\mathbf{J}_{s} .
\end{aligned}
$$

The resulting difference equations for the electric and magnetic field components are given in [23] by applying the 
total-field/scattered field formulation. The back-scattered fields for each source region are computed by FDTD at $S_{c}^{-}$(the closed surface exterior to, and surrounding, $S_{c}$ ). The equivalent surface currents due to these fields, representing an additional source in the MoM domain are given by

$$
\begin{aligned}
\mathbf{J}_{i b} & =-\hat{\mathbf{n}} \times \mathbf{H}_{b} \\
\mathbf{M}_{i b} & =\hat{\mathbf{n}} \times \mathbf{E}_{b}
\end{aligned}
$$

where $\mathbf{H}_{b}$ and $\mathbf{E}_{b}$ are the back-scattered fields computed at $S_{c}^{-}$. $\mathbf{J}_{i b}$ and $\mathbf{M}_{i b}$ are the electric and magnetic equivalent surface currents at $S_{c}^{-}$. Now, the voltage back-scattered on the source region (the excitation for the MoM) can be evaluated using either of the following equations defined by the reciprocity theorem:

$$
\begin{aligned}
V_{b} & =\int_{S_{a}} \mathbf{J}_{t s} \bullet \mathbf{E}_{i b} d s_{a} \\
V_{b} & =\int_{S_{c}^{-}}\left(\mathbf{E}_{t s} \bullet \mathbf{J}_{i b}-\mathbf{H}_{t s} \bullet \mathbf{M}_{i b}\right) d s_{c}^{-}
\end{aligned}
$$

where

$$
\begin{array}{ll}
\mathbf{E}_{i b} & =-j \omega \mathbf{A}(\mathbf{r})-\nabla V(\mathbf{r})-(1 / \varepsilon) \nabla \times \mathbf{F}(\mathbf{r}) ; \\
\mathbf{A}(\mathbf{r}) & =\mu \int_{S_{c}^{-}} \mathbf{J}_{i b} g\left(\mathbf{r}, \mathbf{r}^{\prime}\right) d s_{c}^{-} ; \\
V(\mathbf{r}) & =(-j / \omega \varepsilon) \int_{S_{c}^{-}} \nabla_{s}^{\prime} \bullet \mathbf{J}_{i b} g\left(\mathbf{r}, \mathbf{r}^{\prime}\right) d s_{c}^{-} ; \\
\mathbf{F}(\mathbf{r}) & =\varepsilon \int_{S_{c}^{-}} \mathbf{M}_{i b} g\left(\mathbf{r}, \mathbf{r}^{\prime}\right) d s_{c}^{-} ; \\
g\left(\mathbf{r}, \mathbf{r}^{\prime}\right) & =e^{-j k\left|\mathbf{r}-\mathbf{r}^{\prime}\right| / 4 \pi\left|\mathbf{r}-\mathbf{r}^{\prime}\right| \text { is the free space }} \\
& \text { Green's function. }
\end{array}
$$

The vectors $\mathbf{r}$ and $\mathbf{r}^{\prime}$ apply to the source and observation points respectively. $S_{a}$ is the conducting surface area of the antenna structure. $\mathbf{J}_{t s}$ is the electric test function used on the wire or the patch, usually a uniform pulse to account for the excitation voltage at the center of the wire segment and the patch for the MoM using NEC [24]. $\mathbf{E}_{t s}$ and $\mathbf{H}_{t s}$ are the electric and magnetic fields respectively for the test function $\mathbf{J}_{t s}$. It should be noted that, in applying (7), there is no magnetic test function specified in NEC [24]. Since $\mathbf{E}_{t s}$ and $\mathbf{H}_{t s}$ can be obtained easily using NEC, after ignoring the sinusoidal basis function, and noting that the cell mesh size used in FDTD will be very small compared to the operating wavelength, (8) can be reduced by ignoring the surface integral and evaluating the voltage back-scattered corresponding to the center of the cell surface, as shown below

$$
V_{b}=\sum_{n_{s_{\bar{c}}}}\left(\mathbf{E}_{t s}\left(\mathbf{r}, \mathbf{r}_{n}^{\prime}\right) \bullet \mathbf{J}_{i b_{n}}-\mathbf{H}_{t s}\left(\mathbf{r}, \mathbf{r}_{n}^{\prime}\right) \bullet \mathbf{M}_{i b_{n}}\right) a_{n}
$$

where $\mathbf{r}_{n}^{\prime}$ is the position vector of the center of the cell surface $S_{c}^{-}, n_{s_{c}^{-}}$is the total number of the cell surfaces on the equivalent surface $S_{c}^{-}$and $a_{n}$ is the surface area of the cell. Therefore $\mathbf{J}_{i b n}$ and $\mathbf{M}_{i b n}$ are considered to be the equivalent surface currents at the center of the surface of the cell $n$. Since the excitation voltages are known, the MoM can be executed to compute the new currents and the procedure can be repeated until a steady state solution is reached. A flowchart of the procedure is shown in Fig. 2.

\section{TeStS OF THE METHOD}

As a first step, a test with a strongly coupled structure was undertaken to check the correct functioning of the total/scattered field formulation of the hybrid code. It also served to test the

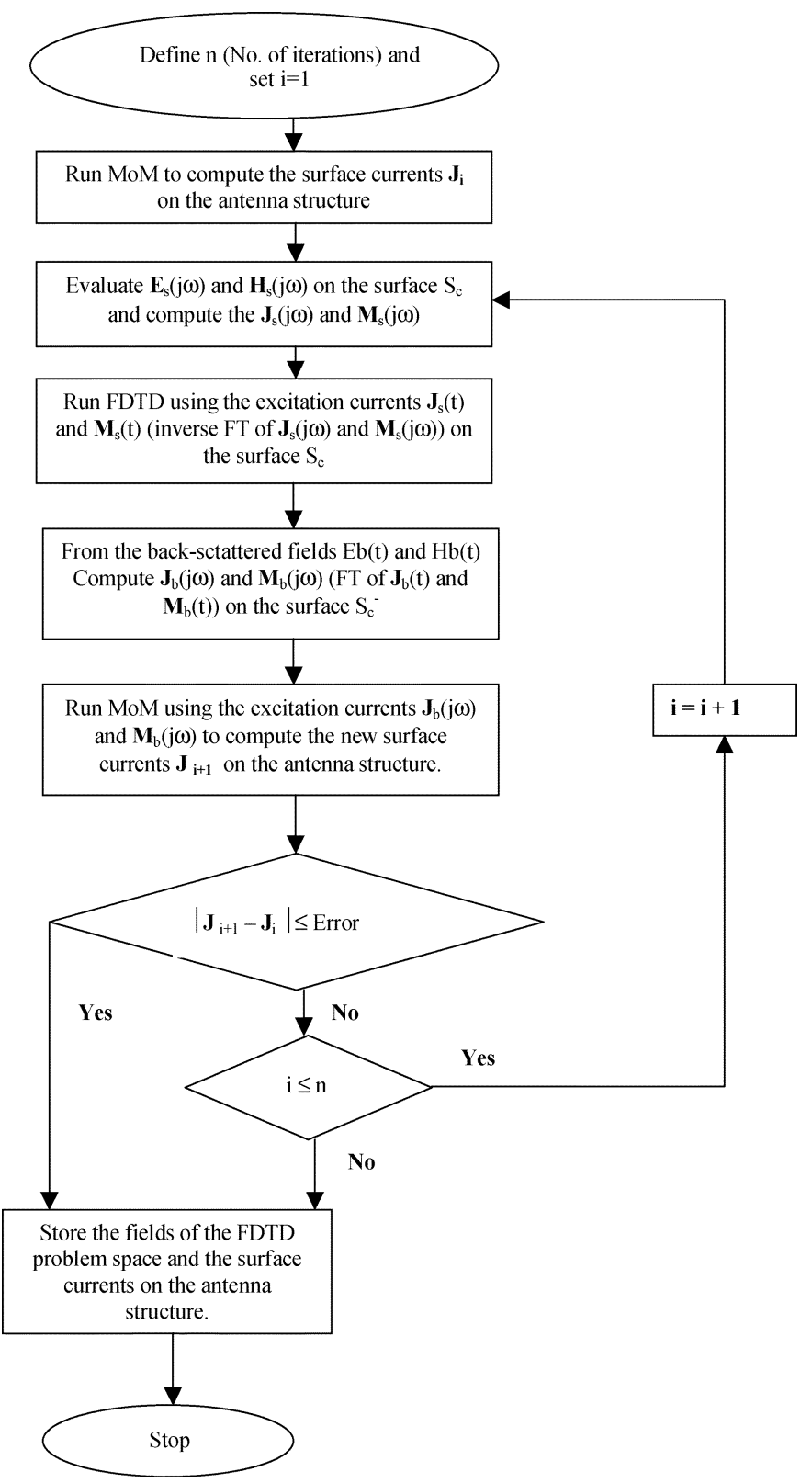

Fig. 2. Flow chart for the MoM/FDTD hybrid method algorithm.

number of iterations required for convergence and the effect of the Huygens surface size. In a second test a dipole antenna was modeled close to a human head, but exterior to the FDTD region modeling the fields in the head. The results were compared with those from a purely FDTD computation, using a larger region that enclosed the dipole.

\section{A. Dipole Adjacent to Perfectly Conducting Plate}

An antenna adjacent to a perfectly conducting plate was first chosen as a verification example. The antenna was modeled using both NEC [24] and an in-house MoM code (implementing polynomial basis functions and Galerkin's solution [17]): it was a half-wavelength dipole with wire radius $0.006 \lambda$, directed along the $z$-axis. The conducting plate used had height $=3 / 2 \lambda$ and width $=\lambda$, modeled by FDTD exactly as for the verification example of the time-domain MoM/FDTD technique used 


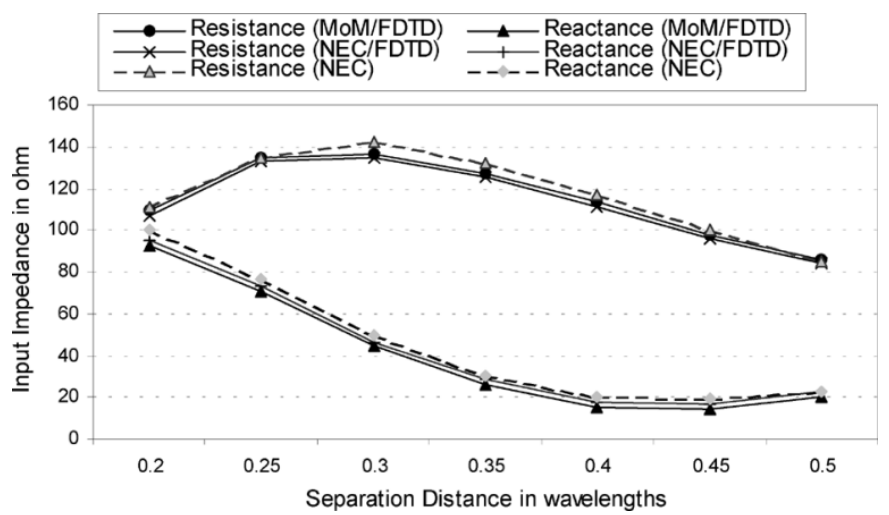

Fig. 3. Input impedance of a dipole adjacent to a plate versus separation distance in wavelengths for both versions of hybrid code, compared with NEC.

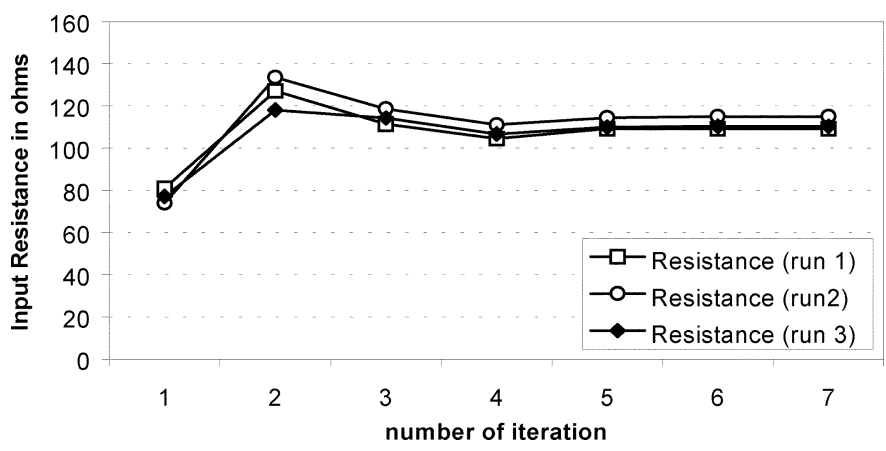

Fig. 4. Input resistance versus number of iterations for $0.2 \lambda$ separation between dipole and plate, for the three runs of example given in Section III-A.

in [8]. The separation distance between the antenna and the plate was varied and the input impedance of the dipole was the target of comparisons between a pure NEC model [24] and both hybrid code versions (in-house MoM/FDTD [20] and NEC/FDTD [17]).

Three runs were executed for different Huygens surface sizes: "run 1 " - a $20 \times 6 \times 60$ cell Huygens surface surrounding the antenna; "run 2 " - an $80 \times 12 \times 80$ cell Huygens surface surrounding the plate; "run 3 " - a $100 \times 10 \times 100$ cell Huygens surface surrounding the plate. The FDTD cell size was $0.02 \lambda$.

Fig. 3 shows the input impedance of the dipole versus separation distance from the plate, for the "run 2" case. Very good agreement is observed between the two versions of the hybrid code and the NEC model for the same problem. The convergence rate is illustrated in Figs. 4 and 5 which show that four or five iterations are sufficient, even for a modest $(0.2 \lambda)$ separation between dipole and plate. This is a remarkably low number of iterations: for a highly resonant structure it could be higher, while for more heavily damped structures (e.g., biological tissue) the number could be lower, as it depends on the fraction of backscattered field with respect to the forward field.

\section{B. Validation Test With Human Head Model (Pure FDTD versus Hybrid)}

The test simulated a dipole antenna close to a human head, corresponding to the situation of a person close to a microcell antenna located at face level. The antenna was excited at $1800 \mathrm{MHz}$, with parameters: length $(\lambda / 2)=0.083 \mathrm{~m}$; radius $=$ $0.00125 \mathrm{~m}$; distance $(D)$ from the centre of the antenna to the

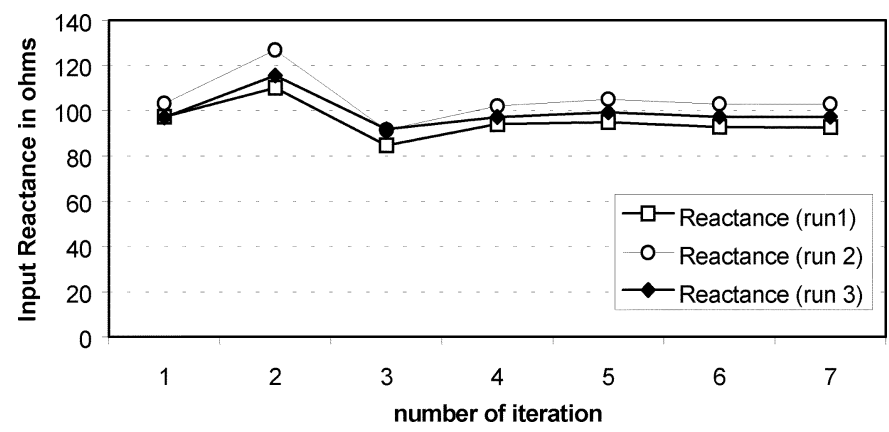

Fig. 5. Input reactance for the case in Fig. 4.

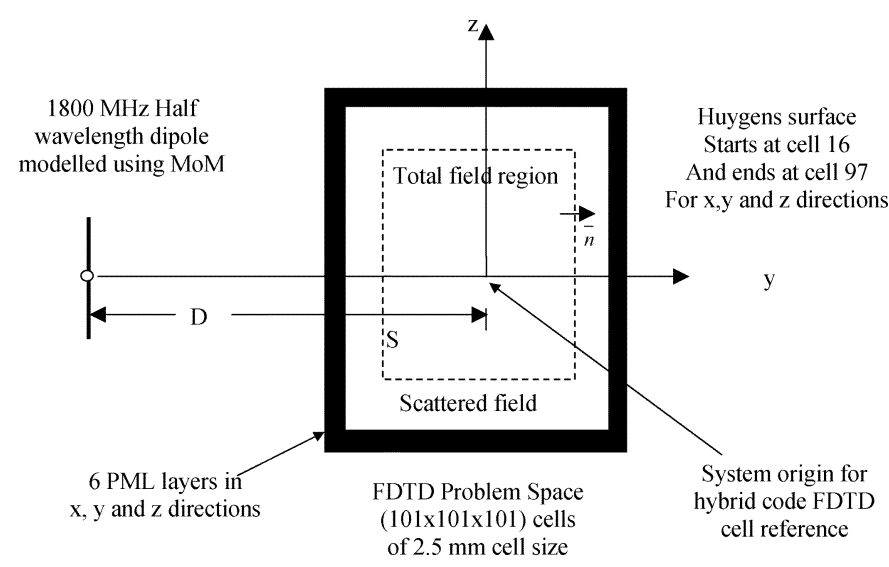

Fig. 6. Hybrid MoM/FDTD problem space for the example given in Section III-B.

origin (the centre of the head $)=0.245 \mathrm{~m}(\sim 3 \lambda / 2)$; number of segments in MoM model of dipole $=31$ (see Fig. 6). The existing Bradford University human head model was used as a test piece [25]: this is clearly insufficient to model whole-body interaction with base station fields, but was adequate to test the method. The head was located with respect to the $x, y$, and $z$ axes as follows:

- $\quad x$ axis is considered to be the ear-to-ear line;

- $y$ axis is considered to be passing through the largest distance $(17 \mathrm{~cm})$ from the back to the front of the head (the nose axis) and the origin is located on the centre of this line;

- $\quad z$ axis is vertical, through the origin.

The system was modeled in two ways: a) with the hybrid method, so that the FDTD region only enclosed the head, and b) with a purely FDTD method, enclosing the head and the dipole. It was essential that the dipole be located relatively close to the head, so that a fully-FDTD model would be tractable, using current computers.

For case a), the FDTD zone parameters were: cell size = $2.5 \mathrm{~mm}$; problem space size (including PML ABC) $=112 \times$ $112 \times 112$ cells; Huygens surface size $=81 \times 81 \times 81$ cells (centred); number of cycles of waveform used in each update of the FDTD region $=25$; number of time steps per cycle $=178$. For case b), the antenna was modeled in FDTD by setting the electric field parallel to its axis equal to zero along its length, which was $8.25 \mathrm{~cm}$ (33 cells). The radius of the antenna was not taken into account in this pure FDTD simulation: the problem space size was $112 \times 152 \times 112$ cells. 


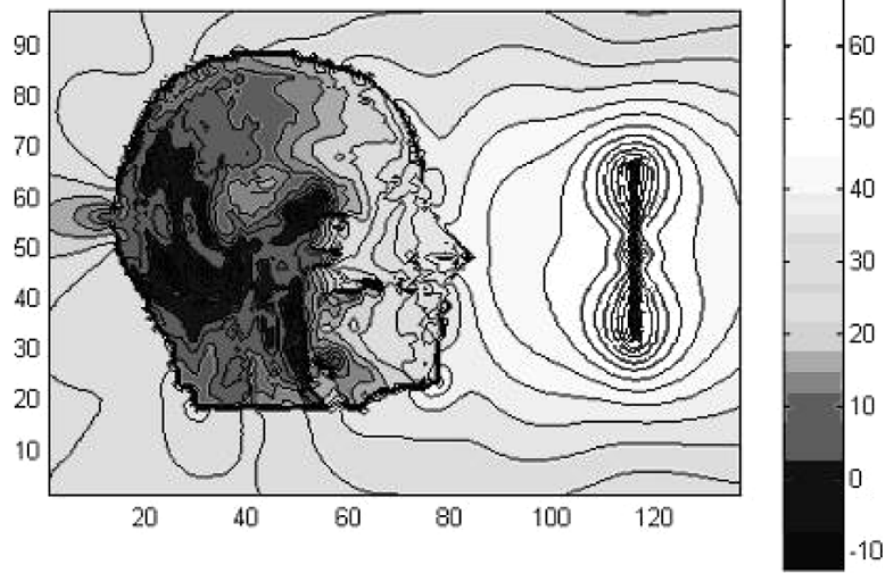

Fig. 7. Electric field magnitude over the central $x-z$ slice of the head-dipole system, using pure FDTD code normalized to $1 \mathrm{~W}$ antenna radiated power at $1800 \mathrm{MHz}$ (scale is in decibels).

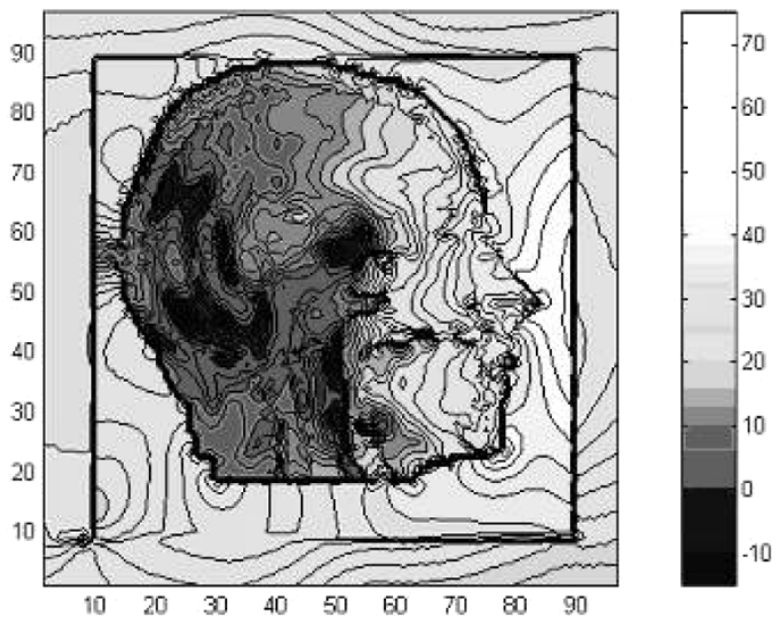

Fig. 8. Electric field magnitude over the central $x-z$ slice, calculated using hybrid code for the situation in Fig. 7. The antenna is outside the displayed (FDTD) zone, to the right.

Figs. 7 and 8 show a comparison of the electric field magnitude over the central $x-z$ slice. The antenna radiated power was normalized to $1 \mathrm{~W}$ and the scale shown is in decibels. It is clear that the distributions within the head and in its near environment compare very well. The electric field distributions were converted to SAR and averaged over $10 \mathrm{~g}$ (as specified in the NRPB and ICNIRP standards [1], [2]): results are shown in Table I.

The maximum difference in the absolute SAR values, averaged over $10 \mathrm{~g}$, computed by the pure FDTD method and the hybrid method was 0.8031 versus 0.7684 , equivalent to $4.32 \%$. This is a satisfactory result, particularly since the representation of the dipole in the hybrid method is much more accurate than in pure FDTD.

\section{STUdies of SAR in a HeAd Close to A MiCROCELL ANTENNA}

The situation modeled in case (b) above was used to investigate the safety compliance of low-power microcell base sta-
TABLE I

SAR AND POWER RESUlTS FOR $\lambda / 2$ DiPOLE RADIATING $1 \mathrm{~W}$ AT $1800 \mathrm{MHz}$ CAlCulated Using PuRe FDTD and Hybrid Codes. THE ANTENNA IS $0.5 \lambda$ FROM THE FRONT OF THE HEAD

\begin{tabular}{l|l|l|l}
\hline Method & $\begin{array}{l}\text { Absorbed } \\
\text { power in } \\
\text { head, } \mathbf{W}\end{array}$ & $\begin{array}{l}\text { SAR (peak), } \\
\text { (W/kg) }\end{array}$ & $\begin{array}{l}\text { Max. SAR } \\
\text { (10g ave.) } \\
\text { W/kg }\end{array}$ \\
\hline Pure FDTD & 0.0821 & 6.8673 & 0.8031 \\
\hline Hybrid & 0.0768 & 6.6276 & 0.7684 \\
\hline
\end{tabular}
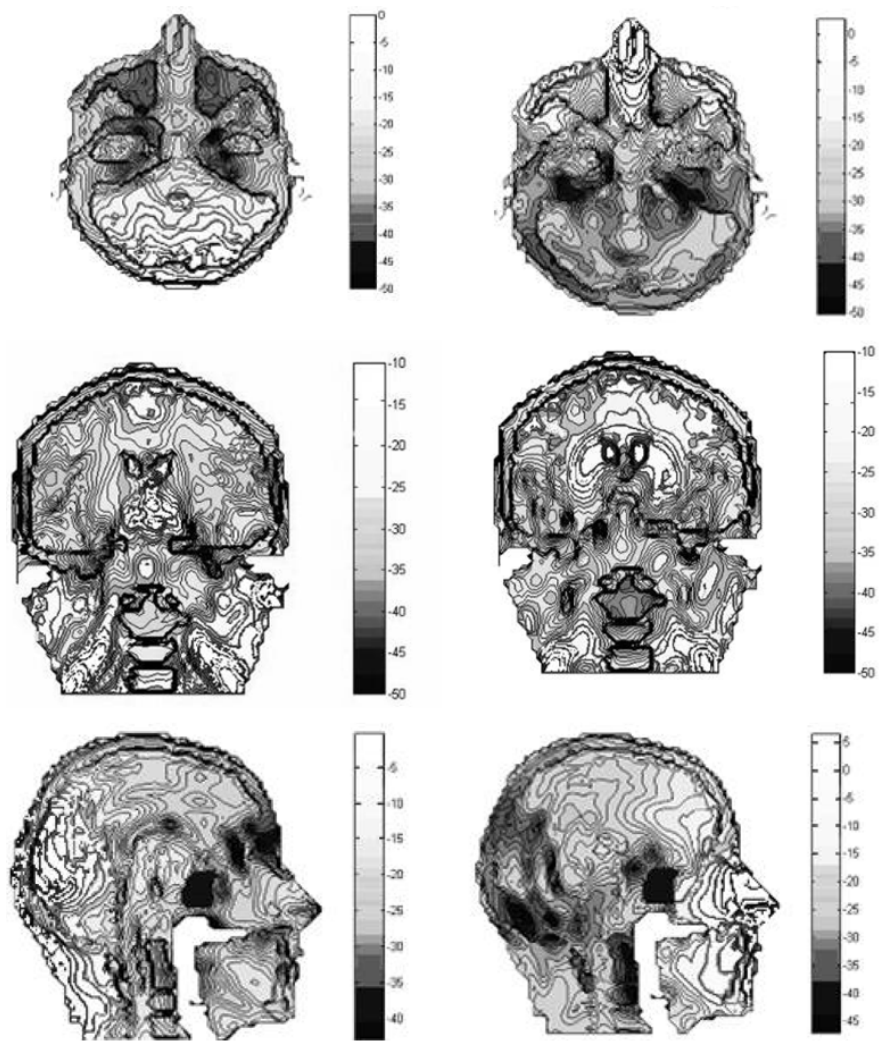

Fig. 9. Peak SAR calculations (in deciebls) for the three main median planes for half wavelength dipole antenna behind (left column) and in front of (right column) the head. Frequency $1800 \mathrm{MHz}$, distance $0.5 \lambda$ from nearest point of head. Radiated power normalized to $1 \mathrm{~W}$.

tion antennas very close to the head. At small distances, it is reasonable to omit the rest of the body from the model: this enables computer power to be focused on high-resolution modeling of the head structure. Six simulation runs were undertaken at $1800 \mathrm{MHz}$, with the antenna $0.5,1$, and 2 wavelengths from the head, which was oriented facing both toward, and away from, the antenna. The SAR values are shown in Table II and the SAR distributions in Fig. 9 (different sections for same distance) and Fig. 10 (same section for different distances). Similarly, the results for $900 \mathrm{MHz}$ are shown in Table III and Fig. 11.

The results in Table II show that, for a radiated power of $1 \mathrm{~W}$, the SAR is well within the ICNIRP guidelines for the particular case presented here; however it is noteworthy that, when averaged over $10 \mathrm{~g}$, the maximum SAR with the antenna in front of the head is higher than when it is at the rear. The graphs in 

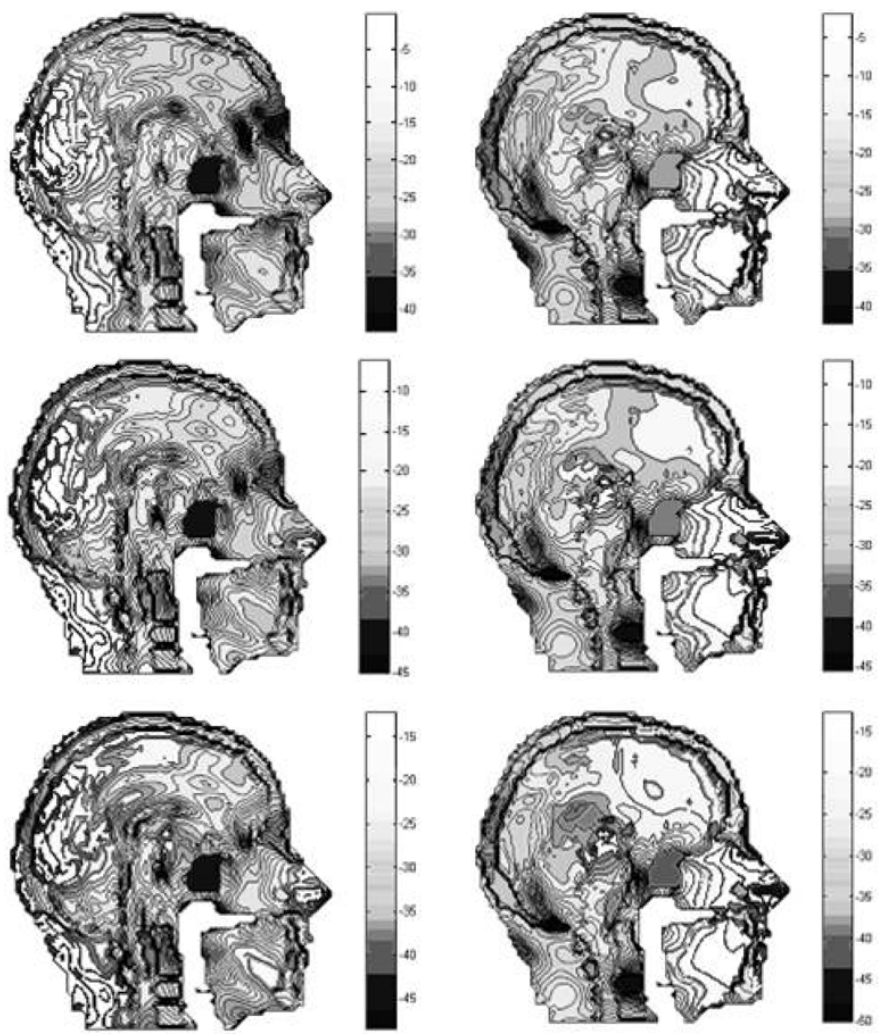

Fig. 10. Peak SAR calculations (in decibels) for front-rear vertical median plane for half wavelength dipole antenna behind (left column) and in front of (right column) the head, for the distances shown. Frequency $1800 \mathrm{MHz}$, radiated power normalized to $1 \mathrm{~W}$.

Fig. 9 appear to show that this is because of local field concentration in the nose. On the other hand, when the SAR is averaged over the whole head (analogous to the "whole-body" averaging threshold stipulated in many standards), the situation with the antenna at the rear is slightly more onerous. The results in Fig. 10 show the expected fall in maximum SAR and a trend toward more uniform distribution of SAR across the cross section with increasing distance from the antenna. In particular, it may be noted that the SAR in the nose becomes relatively intense even when the antenna is at the rear of the head, when it is at the largest distance investigated ( 2 wavelengths): this is presumably due to the phenomenon of field concentration at a point.

For the $900-\mathrm{MHz}$ case, it is clear from the results that the worst cases are again, for $10 \mathrm{~g}$ averaging, when the antenna is in front of the head and, for whole-head averaging, when it is at the back. The appropriate worst cases will thus be used for calculating maximum power limits for comparison with the results from the various PFD methods for safety-compliance assessment. The results in Fig. 11 show the same phenomena as observed in Fig. 10, but with an increased tendency toward uniformity, partly due to the greater penetration depth at this lower frequency and partly due to the greater distances from the antenna, since the distances were measured in wavelengths.

\section{MAXIMUm PeRmissible RADiATEd PoWers Dictated by THE SAR METHOD}

By dividing the values of maximum SAR in Tables II and VI by the limiting values specified in the safety guidelines, the
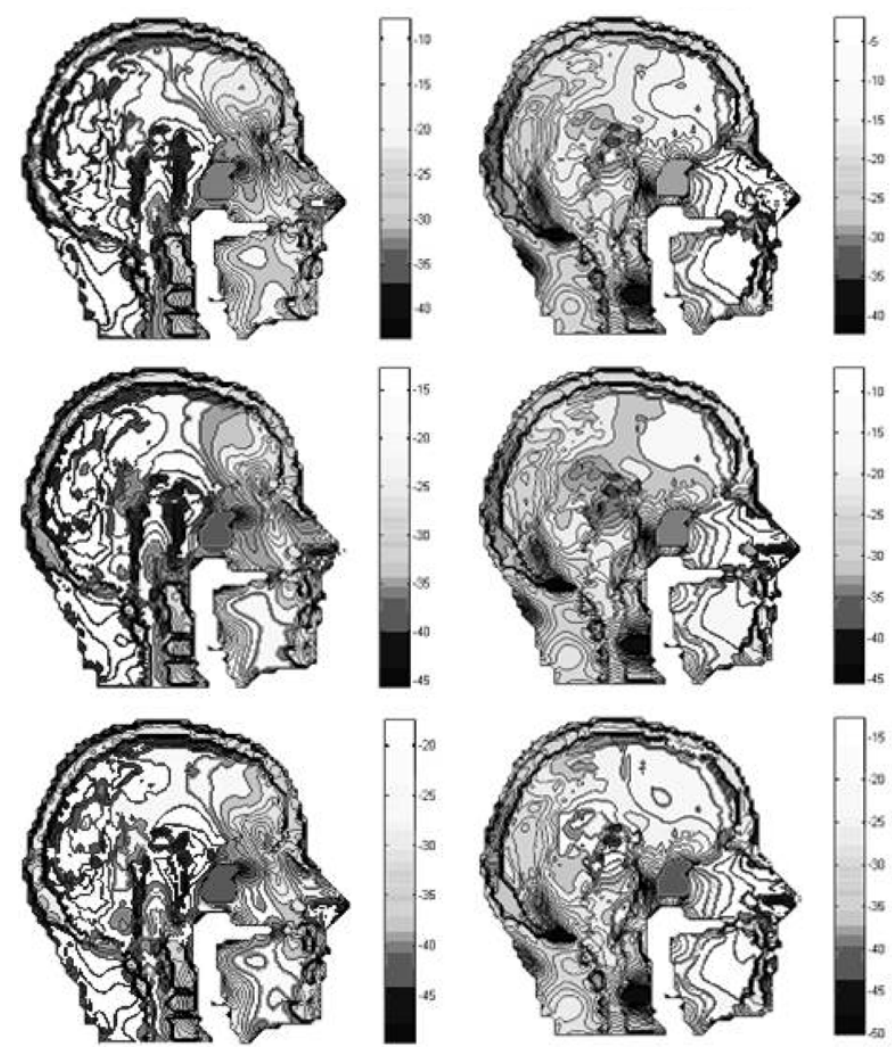

Fig. 11. Peak SAR calculations (in decibels) for front-rear vertical median plane for half wavelength dipole antenna behind (left column) and in front of (right column) the head, for the distances shown. Frequency $900 \mathrm{MHz}$, radiated power normalized to $1 \mathrm{~W}$.

TABLE II

SAR AND POWER Results for $\lambda / 2$ Dipole Radiating $1 \mathrm{~W}$ at $1800 \mathrm{MHz}$

\begin{tabular}{l|l|l|l|l|l}
\hline \multirow{2}{*}{$1800 \mathrm{MHz}$} & $\begin{array}{l}\text { Distance from } \\
\text { head surface in } \\
\text { wavelengths }\end{array}$ & $\begin{array}{l}\text { Absorbed } \\
\text { power in } \\
\text { head, W }\end{array}$ & $\begin{array}{l}\text { SAR } \\
\text { averaged } \\
\text { over whole } \\
\text { head, W/kg }\end{array}$ & $\begin{array}{l}\text { SAR } \\
\text { (peak), } \\
\text { W/kg }\end{array}$ & $\begin{array}{l}\text { Max. SAR } \\
\text { (10g ave.), } \\
\text { W/kg }\end{array}$ \\
\hline $\begin{array}{l}\text { Antenna in } \\
\text { front of } \\
\text { the head }\end{array}$ & 0.5 & 0.0768 & 0.025927 & 6.6276 & 0.7684 \\
\cline { 2 - 6 } & 1 & 0.0373 & 0.012592 & 2.0006 & 0.2424 \\
\hline $\begin{array}{l}\text { Antenna at } \\
\text { the back of } \\
\text { the head }\end{array}$ & 0.5 & 0.0145 & 0.004895 & 0.5659 & 0.0697 \\
\cline { 2 - 6 } & 1 & 0.0888 & 0.029978 & 1.5281 & 0.3510 \\
\hline
\end{tabular}

TABLE III

SAR AND POWER Results For $\lambda / 2$ Dipole RAdiating $1 \mathrm{~W}$ AT $900 \mathrm{MHZ}$

\begin{tabular}{l|l|l|l|l|l}
\hline \multirow{2}{*}{$\mathbf{9 0 0 M H z}$} & $\begin{array}{l}\text { Distance from } \\
\text { head surface in } \\
\text { wavelengths }\end{array}$ & $\begin{array}{l}\text { Absorbed } \\
\text { power, W }\end{array}$ & $\begin{array}{l}\text { SAR averaged } \\
\text { over whole } \\
\text { head, W/kg }\end{array}$ & $\begin{array}{l}\text { SAR } \\
\text { (peak), } \\
\text { W/kg }\end{array}$ & $\begin{array}{l}\text { Max. SAR } \\
\text { (10g ave.), } \\
\mathbf{W} / \mathbf{k g}\end{array}$ \\
\hline \multirow{2}{*}{$\begin{array}{l}\text { Antenna in } \\
\text { front of } \\
\text { the head }\end{array}$} & 0.5 & 0.0568 & 0.019175 & 0.8891 & 0.1542 \\
\cline { 2 - 7 } & 1 & 0.0224 & 0.007562 & 0.2670 & 0.0458 \\
\hline $\begin{array}{l}\text { Antenna in } \\
\text { the back } \\
\text { of the } \\
\text { head }\end{array}$ & 0.5 & 0.0072 & 0.002431 & 0.0720 & 0.0123 \\
\cline { 2 - 6 } & 1 & 0.0591 & 0.019952 & 0.2494 & 0.0924 \\
\cline { 2 - 7 } & 2 & 0.0232 & 0.007832 & 0.0726 & 0.0270 \\
\hline
\end{tabular}

maximum power that may be radiated by the dipole may be calculated (Table IV). Considering the data for $1800 \mathrm{MHz}$, it is noticeable that the values generated by whole-body averaging are below the corresponding ones for $10 \mathrm{~g}$ averaging, except at the shortest distance. This indicates that the SAR is relatively diffuse at distances greater than a half-wavelength at this frequency 
TABLE IV

MAXIMUM POWERS THAT MAY BE FED TO THE DIPOLE

\begin{tabular}{l|l|l|l}
\hline $\mathbf{1 8 0 0}$ MHz: distance & $0.5 \lambda$ & $\lambda$ & $2 \lambda$ \\
\hline NRPB Limits (10g) & $13.0 \mathrm{~W}$ & $41.3 \mathrm{~W}$ & $143 \mathrm{~W}$ \\
\hline ICNIRP Limits (10g) & $2.60 \mathrm{~W}$ & $8.25 \mathrm{~W}$ & $28.7 \mathrm{~W}$ \\
\hline NRPB Limits (WB) & $13.3 \mathrm{~W}$ & $27.6 \mathrm{~W}$ & $72.2 \mathrm{~W}$ \\
\hline ICNIRP Limits (WB) & $2.67 \mathrm{~W}$ & $5.52 \mathrm{~W}$ & $14.4 \mathrm{~W}$ \\
\hline $\mathbf{9 0 0}$ MHz: distance & $0.5 \lambda$ & $\lambda$ & $2 \lambda$ \\
\hline NRPB Limits (10g) & $64.9 \mathrm{~W}$ & $218 \mathrm{~W}$ & $813 \mathrm{~W}$ \\
\hline ICNIRP Limits (10g) & $13.0 \mathrm{~W}$ & $43.7 \mathrm{~W}$ & $163 \mathrm{~W}$ \\
\hline NRPB Limits (WB) & $20.0 \mathrm{~W}$ & $51.1 \mathrm{~W}$ & $158 \mathrm{~W}$ \\
\hline ICNIRP Limits (WB) & $4.01 \mathrm{~W}$ & $10.2 \mathrm{~W}$ & $31.6 \mathrm{~W}$ \\
\hline WB
\end{tabular}

$\mathrm{WB}=$ Whole body averaging (confined to head in this case)

$10 \mathrm{~g}=10 \mathrm{~g}$ averaging

TABLE V

MAXIMUM PERMisSiBLE POWERS AT $1800 \mathrm{MHZ}$

\begin{tabular}{l|l|l|l}
\hline Authority & Method & $\begin{array}{l}\text { Max. power at } \mathbf{0 . 0 8 3 m} \\
(\mathbf{W})\end{array}$ & $\begin{array}{l}\text { Max. power at } \mathbf{0 . 3 3 m} \\
(\mathbf{W})\end{array}$ \\
\hline \multirow{2}{*}{ NRPB } & PFD & 5.54 & 78.2 \\
\cline { 2 - 4 } & SAR & $13.0(10 \mathrm{~g})$ & $72.2(\mathrm{WB})$ \\
\hline \multirow{2}{*}{$\begin{array}{l}\text { (pNIRP } \\
\text { public) }\end{array}$} & PFD & 0.521 & 7.35 \\
\cline { 2 - 4 } & SAR & $2.60(10 \mathrm{~g})$ & $14.4(\mathrm{WB})$ \\
\hline
\end{tabular}

Note: The SAR-based power is whichever is given by the lower of the two averaging methods, as indicated in brackets.

TABLE VI

MAXIMUM PERMISSIBLE POWERS AT $900 \mathrm{MHz}$

\begin{tabular}{l|l|l|l}
\hline Authority & Method & $\begin{array}{l}\text { Max. power at } \\
\mathbf{0 . 1 6 6 m}(\mathbf{W})\end{array}$ & $\begin{array}{l}\text { Max. power at } \mathbf{0 . 6 6 m} \\
(\mathbf{W})\end{array}$ \\
\hline \multirow{2}{*}{ NRPB } & PFD & 8.00 & 113 \\
\cline { 2 - 4 } & SAR & $20.0(\mathrm{WB})$ & $158(\mathrm{WB})$ \\
\hline \multirow{2}{*}{ ICNIRP (public) } & PFD & 1.01 & 14.3 \\
\cline { 2 - 4 } & SAR & $4.01(\mathrm{WB})$ & $31.6(\mathrm{WB})$ \\
\hline
\end{tabular}

Note: The SAR-based power is whichever is given by the lower of the two averaging methods, as indicated in brackets, however this is always whole-body averaging for this frequency and these distances.

and the whole-body criterion takes precedence in this region. At distances of a half-wavelength and less, the $1800-\mathrm{MHz}$ values generated by $10 \mathrm{~g}$ averaging pass below those for whole-body averaging and will clearly become more significant at shorter distances. At $900 \mathrm{MHz}$, it is seen that the whole-body SAR values are well below those for $10 \mathrm{~g}$ averaging, even at $\lambda / 2$ distance.

\section{COMPARISON OF OUTCOMES OF SAR AND PFD METHODS}

The implications of SAR and PFD-based assessment for $1800 \mathrm{MHz}$ can be succinctly compared, at the limits of the distance range studied, in Table V. The NRPB criteria at $0.33 \mathrm{~m}$ show a single case where SAR-based assessment leads to a lower value of maximum power than PFD-based, but the difference is small. For all of the other cases shown, use of SAR-based assessment gives a higher value of permitted maximum power, by factors of between two and five.

Considering now the corresponding results for $900 \mathrm{MHz}$, it is seen that the results generated by whole-body averaging stay well below the corresponding ones for $10 \mathrm{~g}$ averaging at all of the distances examined. This suggests that "peaking" of the SAR is not significant at the distances displayed here.

The implications of SAR and PFD-based assessment at $900 \mathrm{MHz}$ are compared in Table VI. In all of these cases,
SAR-based assessment leads to a higher value of maximum power than PFD-based, by factors of between 1.4 and 4 .

Regarding the contents of the Tables III-VI, it is evident that the PFD criteria, being based on approximations, contain conservative estimates that restrict the maximum allowable powers, in most cases, to a lower level than those permitted by the SAR method. In almost all cases, the SAR method permits the radiation of more power than is allowed by the PFD method. The most significant cases are seen when the ICNIRP guidelines are compared: the power allowed by the SAR method is between two and five times greater than that allowed by the PFD method. Since the SAR method is based on more precise science, it should be taken as the more accurate guide.

\section{CONCLUSION}

A new hybrid computational electromagnetics procedure has been presented, implementing an FDTD region as a subdomain within a region modeled by a frequency-domain Method-ofMoments region. The method has been shown to give satisfactory results for test cases involving strong coupling between an antenna and either a conducting plate or a detailed penetrable model of the human head.

The use of the method to verify safety compliance of microcell base station antennas was demonstrated and it has been argued that, since this permits SAR to be determined for antennas at an arbitrary distance, it is superior to the traditional PFD approach for determining safety compliance of relatively distant antennas. The example given has proved the validity of the principle, although a whole-body model would obviously be necessary if more distant antennas were to be considered. It has been shown that the PFD criteria, being based on approximations, contain conservative estimates that restrict the maximum allowable powers. In most cases, the SAR method permits the radiation of more power than is allowed by the PFD method. The most significant cases are seen when the ICNIRP guidelines are compared: the power allowed by the SAR method is between two and five times greater than that allowed by the PFD method. Since the SAR method is based on more precise science, it should be taken as the more accurate guide.

\section{REFERENCES}

[1] A. F. McKinley, S. G. Allen, P. J. Dimbylow, C. R. Murihead, and R. D. Saunders, "Board statement on restrictions on human exposure to static and time varying electromagnetic fields and radiation," Documents of the $N R P B$, vol. 4 , no. $5,1993$.

[2] International Commission on Nonionising Radiation Protection (ICNIRP), "Guidelines for limiting exposure to time-varying electric, magnetic and electromagnetic fields (up to $300 \mathrm{GHz}$," Health Phys., vol. 74, no. 4, pp. 494-522, 1998.

[3] P. Bernadi, "Electromagnetic Dosimetry for Mobile Communication Equipment (Short Title: CEPHOS - Cellular Phone Standards)," EU Standards, Measurements and Testing Programme, Contract no. SMT4-CT97-2173, 1999.

[4] A. Taflove and K. R. Umashankar, "A hybrid moment method/finite difference time-domain approach to electromagnetic coupling and aperture penetration into complex geometries," IEEE Trans. Antennas Propagat., vol. AP-30, no. 4, pp. 617-627, Jul. 1982.

[5] K. R. Umashankar, A. Taflove, and B. Beker, "Calculation and experimental validation of induced currents on coupled wires in an arbitrary shape cavity," IEEE Trans. Antennas Propagat., vol. AP-35, no. 11, pp. 1248-1257, Nov. 1987. 
[6] E. L. Coffey, "Recent enhancments to GEMACS 5.2," in Proc. 9th Annu. Rev. Progress in Applied Computational Electromagnetics, Monterey, CA, 1993, pp. 894-900.

[7] A. R. Bretones, R. Mittra, and G. Martin, "A hybrid technique combining the MoM in the time domain and FDTD," IEEE Microw. Guided Wave Lett., vol. 8, no. 8, pp. 281-283, Aug. 1998.

[8] G. Cerri, P. Russo, A. Schiavoni, G. Tribellini, and P. Bielli, "MoMFDTD hybrid technique for analysing scattering problems," Electron. Lett., vol. 34, no. 5, pp. 433-440, 1998.

[9] A. D. Tinniswood, "Time Domain Integral Equations," Ph.D dissertation, University of York, York, U.K., 1996.

[10] Z. Huang, K. Demarest, and R. Plumb, "An FDTD/MoM hybrid technique for modeling complex antenna in the presence of heterogeneous grounds," IEEE Trans. Geosci. Remote Sensing, vol. 37, no. 6, pp. 2692-2698, Jun. 1999.

[11] J. Chen, Z. Feng, and J. Jin, "Numerical simulation of SAR and B1-field inhomogenity of shielded RF coils loaded with human head," IEEE Trans. Biomed. Eng., vol. 45, no. 5, pp. 650-659, May 1998.

[12] E. A. Forgy, W. C. Chew, and J. M. Jin, "A hybrid MoM/FDTD technique for studying human head/antenna interactions," in Proc. IEEE Antennas Propagation Soc. Conf., Boston, MA, 1998, pp. 81-84.

[13] K. A. Lysiak, J. K. Breakall, and J. Zmyslo, "A hybrid MoM/FDTD approach to UHF/VHF propagation problems," in IEEE Antennas and Propagation Soc. Int. Symp., Baltimore, MD, 1996, pp. 358-361.

[14] A. Rubio Bretones, R. Mittra, and R. Gomez Martin, "Hybrid NEC/FDTD approach for analysing electrically short thin-wire antennas located in proximity of inhomogeneous scatterers," Electron. Lett., vol. 35, no. 19, pp. 1594-1596, 1999.

[15] A. D. Monk, M. Rayner, and A. D. Olver, "A comparison of FDTD and method of moments to model electrically small antennas," in IEEE Antennas and Propagation Soc. Int. Symp., vol. 1, 1994, pp. 565-68.

[16] J. S. Colburn, M. A. Jensen, and Y. Rahmat-Samii, "Comparison of MoM and FDTD for radiation and scattering involving dielectric objects'," in IEEE AP Soc. Int. Symp., vol. 1, 1995, pp. 644-47.

[17] M. A. Mangoud, R. A. Abd-Alhameed, and P. S. Excell, "Simulation of human interaction with mobile telephones using hybrid techniques over coupled domains," IEEE Trans. Microw. Theory Tech., vol. 48, no. 11, pp. 2014-2021, Nov. 2000 .

[18] R. A. Abd-Alhameed, P. S. Excell, J. A. Vaul, and M. A. Mangoud, "A hybrid treatment for electromagnetic field computation in multiple regions," Electron. Lett., vol. 34, no. 20, pp. 1925-1926, 1998.

[19] M. A. Mangoud, R. A. Abd-Alhameed, P. S. Excell, and J. A. Vaul, "Conduction current crossing domain boundaries in a heterogeneous hybrid computational electromagnetics formulation," Electron. Lett., vol. 35, no. 21, pp. 1786-1787, 1999.

[20] R. A. Abd-Alhameed, P. S. Excell, J. A. Vaul, and M. A. Mangoud, "Computation of radiated and scattered fields using separate frequencydomain moment-method regions and frequency-domain MoM-FDTD hybrid methods," in Proc. IEE Conf. Antennas and Propagation, York, U.K., 1999, pp. 53-6.

[21] R. A. Abd-Alhameed, P. S. Excell, M. A. Mangoud, and J. A. Vaul, Hybrid Integral Equation/ Finite Difference Method for Computation of Interaction of Mobile Telephone Antenna with the Human Head. Toronto, Canada: URSI General Assembly, 1999.

[22] D. E. Merewether, R. Fisher, and F. W. Smith, "On implementing a numerical Huygens source scheme in a finite difference program to illuminate scattering bodies," IEEE Trans. Nucl. Sci., vol. NS-27, no. 6, pp. 1829-1833, Jun. 1980

[23] A. Taflove, Computational Electrodynamics: The Finite Difference Time Domain Method. Dedham, MA: Artech House, 1995.

[24] G. J. Burke and A. J. Poggio, "Numerical Electromagnetics Code (NEC): Method of Moments," US Naval Ocean Systems Centre, San Diego, CA, Rep. TD116, 1981.

[25] P. Olley and P. S. Excell, "Classification of a high-resolution voxel image of a human head," in Proc. Int. Workshop Voxel Phantom Development, National Radiological Protection Board, 1995, pp. 16-23.

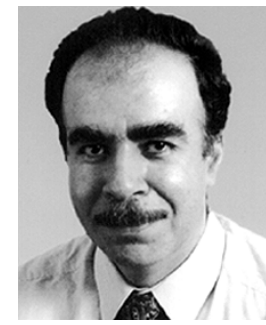

Raed A. Abd-Alhameed was born in Basrah, Iraq in 1959. He received the B.Sc. and M.Sc. degrees from Basrah University, in 1982 and 1985, respectively, and the Ph.D. degree from the University of Bradford, U.K., in 1997, all in electrical engineering.

From 1997 to 1999, he was a Postdoctoral Research Fellow at the University of Bradford, specializing in computational modeling of electromagnetic field problems, microwave nonlinear circuit simulation, signal processing of preadaption filters in adaptive antenna arrays and simulation of active inductance. Since 2000, he has been a Lecturer in the University of Bradford. His current research interests include hybrid electromagnetic computational techniques, antenna design, low SAR antennas for mobile handset, RF mixers. and active antennas.

Dr. Abd-Alhameed is a Member of the Institution of Electrical Engineers, London, U.K. (IEE).

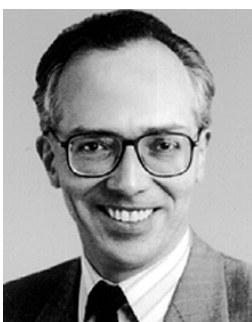

Peter S. Excell (SM'84) obtained the B.Sc. degree from the University of Reading, Reading, U.K., in 1970, and the Ph.D. degree from Bradford University, Bradford, U.K., in 1980, for research in electromagnetic hazards.

$\mathrm{He}$ is a Professor of applied electromagnetics and Deputy Director of the Telecommunications Research Centre at the University of Bradford, U.K., where he has worked since 1971. His research interests include electromagentics since 1974, when he started work on an investigation of hazards due to EM radiation. Later he initiated further research in compact range antennas, computational electromagnetics, bioelectromagnetics, and superconducting filters and antennas. The superconductivity work has concentrated on design of efficient electrically small devices for frequencies below $2 \mathrm{GHz}$. He is a U.K. national representative on the management committee of the European collaboration COST 261 (electromagnetic compatibility in large systems).

Dr. Excell is a Fellow of the Institution of Electrical Engineers, a Chartered Engineer registered with the U.K. Engineering Council, and a Member of the International Union of Radio Science (URSI), the Applied Computational Electromagnetics Society, the Bioelectromagnetics Society, and the European Bioelectromagnetics Association. He is a member of the U.K. national committee of URSI, representing Commission K (electromagnetics in medicine and biology)

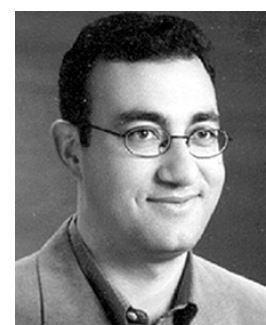

Mohab A. Mangoud (M'01) was born in Alexandria, Egypt, in 1971. He received the B.Sc. and M.Sc. degrees in electrical engineering from Alexandria University, Alexandria, Egypt, in 1993 and 1996, respectively, and the $\mathrm{Ph} . \mathrm{D}$. degree in electrical engineering from the University of Bradford, Bradford, U.K., in 2001.

$\mathrm{He}$ is currently an Assistant Professor in the Electronics and Communications Engineering Department, Arab Academy for Science and Technology and Maritime Transport (AAST), Alexandria, Egypt, where he has worked since 1993. He is responsible for teaching antennas and wireless communications courses. His research interests include modeling of electromagnetic systems, antenna design, and smart antennas. 